\title{
Phase Transitions in Nematic Fluids
}

\author{
A. Oukouiss ${ }^{1,2}$ \\ ${ }^{1}$ Département de Physique, Faculté Pluridisciplinaire - Nador, B.300, 62700, Selouane, Nador, \\ Morroko \\ ${ }^{2}$ Faculté des sciences, Case Postale 231, Université Libre de Bruxelles, B-1050 Bruxelles, Belgium
}

Received 15 February 2010, accepted in revised form 21 July 2010

\begin{abstract}
We use a simple van der Waals theory, suitably extended to the solid phase and to anisotropic interactions, to study the phase behaviour of a system of particles with nematic interactions. Very rich phase behaviour is found which indicates, in particular, that the nematic liquid is stable only for large values of the strength of the nematic interactions. The isotropic liquid-nematic liquid and the isotropic solid-nematic solid transitions are always first-order. Additionally, we have found that the nematic liquid is thermodynamically stable only in a small domain of the temperature-density plane contained between two triple lines.
\end{abstract}

Keywords: Phase diagrams; Nematic interactions; Free energy; Transitions.

(c) 2010 JSR Publications. ISSN: 2070-0237 (Print); 2070-0245 (Online). All rights reserved.

DOI: 10.3329/jsr.v2i3.4438 J. Sci. Res. 2 (3), 419-432 (2010)

\section{Introduction}

The relation between the Hamiltonian of a system and the form of the resulting phase diagram is the central theme of equilibrium statistical mechanics [1,2]. For onecomponent systems with only pair-interaction, to which we will restrict ourselves here, this relation is at present fairly well understood for the particular case of the isotropic interactions which prevail between spherical molecules without embedded (electric or magnetic) dipoles or other anisotropic features [3]. In the simplest case one can usually neatly separate this pair-potential into a harsh repulsive part and a weaker attractive part. Using the overall amplitude of this pair-potential to fix the temperature scale and the range of the repulsions to fix the density scale, the resulting phase diagram can be seen to be largely determined by the characteristic overall features of the attractions [4-6]. This is particularly so with respect to the fluid phases, on which we will focus our attention henceforth, while the solid phases are more sensitive to the finer details of the potential

\footnotetext{
${ }^{1}$ Corresponding author: aoukouiss@yahoo.fr
} 
[7]. In the case of isotropic interactions only two types of fluid phases are possible [8$10,11]$, a low density fluid or vapour phase and a high density fluid or liquid phase. The presence of attractions is a necessary condition for the appearance of a liquid phase in the phase diagram. As has been shown in [4-6], it is, however, not a sufficient condition. Indeed, this liquid phase can still be thermodynamically metastable, i.e., the liquid-vapour transition can still be pre-empted by the fluid-solid transition. This has been shown to be the case whenever the range of the attractions is too short relative to the range of the repulsions [4 -6]. Phase diagrams of systems interacting via density dependent potentials, with a constant excluded volume, can exhibit two phase transitions: gas-liquid and liquidliquid [12-14]. Before one can conclude that a liquid phase will appear in the system's phase diagram it is thus essential to compute the complete phase diagram, including the solid phases, even if one is interested only in the presence of the liquid phase. In the case of anisotropic potentials a similar question can be raised relative to the presence of anisotropic liquids in the phase diagram. To simplify the problem we will consider here situations where only the attractions do exhibit anisotropic features whereas the repulsions remain isotropic. This corresponds to spherical molecules with an embedded anisotropy such as an electric or magnetic dipole.

There are many types of interaction potentials which can be used to describe the interaction between particles of anisotropic fluids.

In this paper we will study the nematic phase using the generalised van der Waals theory $[15,16]$. For this we consider the so-called Maier-Saupe model used to study the formation of liquid-crystalline phases induced by anisotropic repulsions [17].

As is well known, melting from the solid to the liquid is not a single transition, but occurs as a series of transitions into intermediate states called liquid crystal phases. These phases are the states of aggregation that are intermediate between the solid and the liquid. Liquid crystals exhibit a rich variety of phases [18]. One of the simplest (and better understood) is the nematic phase which is caracterized by positional disorder and longrange orientational order.

When the isotropic liquid is cooled the first phase that condenses is the nematic phase in which the orientation of molecules tend to orient in a certain direction so that their orientations are on average parallel to a particular direction specified by a unit vector $\boldsymbol{n}$ called the director. The positions of the molecule's center of mass remain randomly distributed as they are in an isotropic fluid.

If the orientation of each molecule makes an angle $\theta$ with respect to the nematic director $\boldsymbol{n}$, then a measure of the degree of order in the nematic phase is given by [19]

$\frac{1}{2}\left\langle 3 \cos ^{2} \theta-1\right\rangle$, where the angular brackets denote a statistical average.

Systems which exhibit transition to a nematic phase have already been examined by many authors. Onsager [20] did show that particles of a sufficiently anisotropic shape, e.g. long rods or flats discs, form a nematic phase at high densities. Baus et al. [21] have used a density-functional theory for the study of the isotropic-nematic transition of hard ellipsoids. They found that the theory predicts a stable nematic phase, both for rodlike and 
disklike molecules, as a result of competition between the orientational entropy and the anisotropic excluded volume effects. This phase has been confirmed by computer simulations for hard ellipsoids [22] spherocylinders [23-26] and for hard spheres [27]. Recently Mishra et al. [28,29] have used the mean spherical approximation and the percus-Yevick integral equation theories to calculate the free energy functional for the nematic phase. A first-order isotropic-nematic transition is to be found in the liquid regime, although these authors do not present results for the complete phase diagram. They expressed the two-particle density distribution $\rho$ in terms of order parameters and solved the resulting equation for values of order parameters ranging from zero to some maximum value. They constructed a free energy functional and used it to determine the value of order parameters in the nematic phase by minimizing it.

By computer simulation of a system of ellipsoids Phoung and Shmid [30] showed that in the nematic phase there are two qualitatively different contributions: one that preserves rotational invariance and other that breaks it and vanishes in the isotropic phase. Recently, Lomba and al. [31] have used the Monte Carlo computer simulations to study the phase behavior of hard sphere Maier Saupe spins systems. According to this simulation this system undergoes a first-order isotropic-nematic transition continuously coupled to a gasliquid transition.

In our early extensive study of the phase behavior of the Heisenberg model $[15,16]$ we have generalized the van der Waals (vdW) theory for anisotropic interactions to study the phase behavior of a system of particles with magnetic exchange interactions.

By using this vdW theory we have recently developed the calculation of free energy in a nematic fluid phase [32]. We have shown that there is no difficulty in stabilizing the nematic liquid phase over a large domain of the temperature-density plane.

In the present investigation we perform a similar study. We will use the nematic Maier-Saupe interactions [17, 32] and the generalized vdW theory to calculate the free energy functional for the nematic phases (including the solid phases) and examine the thermodynamic stability of nematic liquids when the solid phases are taken into account. The value order parameter has been found by minimization of the reduced Helmholtz free energy functional in terms of order parameter.

This paper is organized as follows. In sec. 2 we introduce our model for the anisotropic potential. The free energy functional for the nematic fluid and solid phases will be computed in sec. 3 from a vdW approximation. The complete phase diagram will be presented in sec. 4 while sec. 5 contains our discussions and conclusions.

\section{The molecular Interactions}

We consider a system of $\mathrm{N}$ identical spherical molecules with an embedded anisotropy (such as an electric or magnetic moment). The position and orientation of these molecules will be specified, respectively, by the coordinates $\left(r_{i}\right)$ of their center of mass and the orientations $\left(s_{i}\right)$ of a unit spin variable $\left(s_{i}^{2}=1\right)$ placed at this center. The pair potential between two such molecules (say, $i=1,2$ ) will be assumed to depend on their relative 
distance, $\left|r_{1}-r_{2}\right|$, and on their relative orientation, $S_{1} \cdot S_{2}$. The interaction potential between two such molecules, $V\left(r_{1}, r_{2}, s_{1} . s_{2}\right)$, will be taken to be of the form :

$$
V\left(r_{1}, r_{2} ; s_{1} \cdot S_{2}\right)=V_{H S}\left(r_{12}\right)+V_{1}\left(r_{12}\right)+V_{M S}\left(r_{12} ; s_{1} \cdot s_{2}\right) \text {, }
$$

where the hard-sphere repulsion $V_{H S}\left(r_{12}\right)$ is given by

$$
V_{H S}\left(r_{12}\right)= \begin{cases}\infty & \text { if } x_{12} \leq 1 \\ 0 & \text { if } \quad x_{12}>1\end{cases}
$$

where $x_{12}=r_{12} / \sigma$ is the reduced distance and $\sigma$ which fixes the range of the repulsion, is the hard-sphere diameter. The second term $V_{1}\left(r_{12}\right)$ of Eq. (1) represents the isotropic (spin-independent) interactions:

$$
V_{1}\left(r_{12}\right)= \begin{cases}0 & \text { if } x_{12}<1 \\ -\varepsilon_{1} \phi_{1}\left(x_{12}\right) & \text { if } x_{12} \geq 1\end{cases}
$$

The third term $V_{M S}\left(r_{12} ; s_{1} \cdot s_{2}\right)$ of Eq. (1) represent the anisotropic (Mayer-Saupe nematic interaction ) interactions :

$$
V_{M S}=V_{2}\left(r_{12}\right) \cdot P_{2}\left(s_{1} \cdot s_{2}\right)
$$

where $P_{2}\left(s_{1} \cdot s_{2}\right)=\frac{1}{2}\left[3\left(s_{1} \cdot s_{2}\right)^{2}-1\right]$ is the Legendre polynomial of order 2 and $V_{2}\left(r_{12}\right)$ is the radial factor of amplitude $\varepsilon_{2}$

$$
V_{2}\left(r_{12}\right)=\left\{\begin{array}{lll}
0 & \text { if } & x_{12}<1 \\
-\varepsilon_{2} \phi_{2}\left(x_{12}\right) & \text { if } & x_{12} \geq 1
\end{array}\right.
$$

The $\phi_{l}(l=1,2)$ in Eqs. (3) and (5) can be taken to be of the inverse-power type [32]:

$$
\phi_{l}\left(x_{12}\right)=1 /\left(x_{12}\right)^{n_{l}}, \quad(l=1,2)
$$

The final pair potential of our Maier-Saupe model can thus written as (see [32] for more details):

$$
V\left(r_{12}\right)= \begin{cases}\infty & \text { if } x_{12}<1 \\ -\varepsilon_{1}\left\{\frac{1}{\left(x_{12}\right)^{n_{1}}}+\frac{1}{2}\left[3\left(s_{1} \cdot s_{2}\right)^{2}-1\right] \frac{\gamma}{\left(x_{12}\right)^{n_{2}}}\right\} & \text { if } x_{12} \geq 1\end{cases}
$$


where $\varepsilon_{1}$ fixes the energy scale and $\gamma=\varepsilon_{2} / \varepsilon_{1}$ measures the strength of the nematic interactions. The reduced potential $V / \varepsilon_{1}$ thus depends on three positive parameters $\left\{\gamma, n_{1}, n_{2}\right\}$.

\section{The free energy}

As already stated above, computing phase diagrams is a very demanding task, certainly for anisotropic potentials such as Eq. (7). In view of this, here we will compute the free energy of the nematic fluids defined by Eq. (7) within a mean-field van der Waals type of approximation which extends the considerations of $[9,10]$ to anisotropic interactions. The resulting theory is very simple and flexible but nevertheless physically realistic when, as here, the major goal is to explore the influence of the potential parameters, such as $\left\{\gamma, n_{1}, n_{2}\right\}$ of Eq. (7), on the topology of the phase diagram.

As is well known, in the vdW-approximation the (hard-sphere) repulsions are described within a free-volume approximation while the attractions are treated within a mean field approximation [9,10,11].

The reduced Helmholtz free energy per particle, $f=F / N \varepsilon_{0}$, of a system with pairpotential of the form defined by Eq. (7) reads (see [32] for more details):

$$
f=t\left[\ln \left(\rho \Lambda^{3}\right)-1\right]-t \ln \alpha(\rho)-\phi_{1}+\gamma \phi_{2} m^{2}-t \ln N(q)
$$

where $t=\frac{k_{B} T}{\varepsilon_{1}}$ is the reduced temperature, $\Lambda$ being the thermal de Broglie wavelength, $\rho=\frac{N}{V}$ the average density and

$$
\begin{aligned}
& q=2 \frac{\gamma}{t} \phi_{2} m, \\
& m=<P_{2}(u)>_{0}=\frac{1}{2} \int_{-1}^{1} d u P_{2}(u) h_{0}(u)=\frac{1}{2}\left(\frac{\exp (q)}{q N(q)}-1-\frac{1}{q}\right), \\
& N(q)=\frac{1}{2} \int_{-1}^{1} d u \exp \left(q P_{2}(u)\right)=\sqrt{\frac{2}{3}} \frac{\exp (q)}{\sqrt{q}} D\left(\sqrt{\frac{3 q}{2}}\right),
\end{aligned}
$$

with $D$ denotes the Dawson function.

$\alpha(\rho)$ in Eq. (8) represents the fraction of the total volume which is freely accessible to the molecules. In particular, we have (see [7,9]

$$
\alpha(\rho)=\left(1-\frac{\rho}{\rho_{0}}\right)
$$

for a fluid phase, with $\left(\frac{\pi}{6}\right) \rho_{0} \sigma^{3}=0.495$ being the packing fraction above which the fluid becomes unstable, and 


$$
\alpha(\rho)=\left[1-\left(\frac{\rho}{\rho_{c p}}\right)^{1 / 3}\right]^{3}
$$

for a solid phase, with $(\pi / 6) \rho_{c p} \sigma^{3}=0.74$ the packing fraction above which a closepacked crystal becomes unstable.

$\phi_{l}(l=1,2)$ in Eq. (8) denotes the following radial average of $\phi_{l}\left(x_{12}\right)$ :

$$
\phi_{l}=\frac{1}{2 N} \int d r_{1} \int d r_{2} \rho\left(r_{1}\right) \phi_{l}\left(\frac{r_{12}}{\sigma}\right) \rho\left(r_{2}\right) \text {. }
$$

with $\phi_{l}\left(\frac{r_{12}}{\sigma}\right)=\phi_{l}\left(x_{12}\right)$ defined by Eq. (3) and (5).

For a fluid phase, $\rho(r) \equiv \rho$, Eq. (14) reduces to the cohesion energy

$$
\phi_{l}=2 \pi \rho \sigma^{3} \int_{1}^{\infty} d x x^{2} \phi_{l}(x)
$$

which, for inverse power potentials of Eq. (6), becomes

$$
\phi_{l}=\frac{2 \pi \rho \sigma^{3}}{n_{l}-3}
$$

For a solid phase (perfect crystal) with lattice $r_{j}$, we have

$$
\rho(r)=\sum_{j=1}^{N} \varphi\left(r-r_{j}\right)
$$

where $\varphi\left(r-r_{j}\right)$ describes the normalized $\left(\int d r \varphi(r)=1\right)$ density profile around the site at $r_{j}$. As in the hard-sphere crystals the particles are strongly localised, we can approximate the density profile $\varphi\left(r-r_{j}\right)$ of Eq. (17) by a delta function. In this case Eq. (17) becomes

$$
\rho(r)=\sum_{j=1}^{N} \delta\left(r-r_{j}\right)
$$

By using Eq. (18), Eq. (14) reduces then, for the solid phase, to the lattice energy

$$
\phi_{l}=\frac{1}{2} \sum_{j} \phi_{l}\left(x_{j}\right)
$$

where the sum runs over the lattice sites, $r_{j} / \sigma=x_{j}>1$, of a periodic lattice without defects, $r_{j}$ being the distance of site $j$ to the site at the origin.

For the inverse power potentials of Eq. (6), we have thus

$$
\phi_{l}=\frac{1}{2} \sum_{j=1}^{N} \phi_{l}\left(x_{j}\right)=\frac{1}{2} \sum_{k}^{\text {shells }} n_{k} \phi_{l}\left(x_{k}\right)=\frac{1}{2} \alpha_{n_{l}}\left(\rho / \rho_{c p}\right)^{n_{l / 3}}
$$


where $\alpha_{n_{l}}$ is the Madelung constant [15]:

$$
\alpha_{n_{l}}=\sum_{j}\left(\frac{x_{1}}{x_{j}}\right)^{n_{l}}
$$

with $x_{j} \geq x_{1}$ and $x_{1}$ the reduced nearest neighbour distance $\left(\rho=\rho_{c p} / x_{1}^{3}\right.$ ).

In the following we will choose $m$ as a new order parameter instead of $q$ because $q$ is unbounded $(0 \leq q \leq \infty)$ whereas $m$ is bounded $(0 \leq m \leq 1)$.

According to the values of $m$ we have then either isotropic phases ( $m=0$ ) or nematic phases $(m \neq 0)$.

By plotting the curves, the reduced free-energy per unit volume $\eta f$ versus the reduced density $\eta=\frac{\pi \rho \sigma^{3}}{6}$, we see that this curve can be divided into two branches: $N$ and $N$ ' (see Fig.1.a,b). The $N$ ' branch is the unphysical branch because $\eta f$ is a concave function of $\eta$. The $N$ branch, however, is the physical branch because $\eta f$ is a convex function of $\eta$ (see [32] for more details).
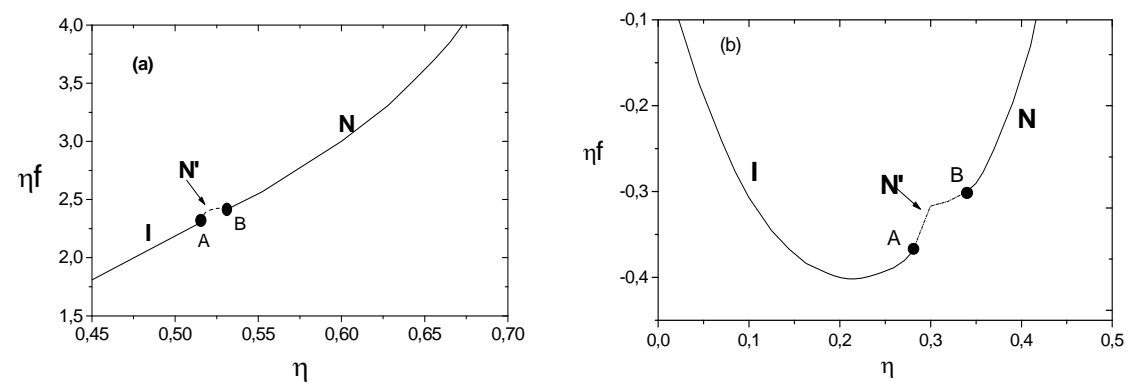

Fig.1. (a,b): Graphical representation of the isotropic (I), unphysical nematic (N') and physical nematic $(\mathrm{N})$ branches of the reduced free energy per unit volume $\eta f$ versus the reduced density $\eta$. The case shown in (b) corresponds to $\Gamma^{*}=\gamma \frac{n_{1}-3}{n_{2}-3}=1.4$ and $t=0.7$ while in (b) corresponds to the solid phases with $n_{1}=n_{2}=6, \gamma=0.8$ and $t=1.3$.

In our previous study [32], we have shown that the nematic phase can only occur for $q$ larger than 1.51 or $\gamma^{*}=\gamma \frac{\phi_{2}}{t}$ larger than 2.24. In other words, the isotropic-nematic transition is always of the first-order type because the order parameter $q$ has to jump from $q=0$ to $q \geq 1.51$.

In the following we will give the phase diagram for the fluid phases (both isotropic and nematic), solid phases, and finally we present the complete phase diagram where both fluid and solid phases are present. 


\section{Phase Diagrams}

For a system of $N$ molecules enclosed in a volume $V$ at the equilibrium temperature $T$, the phase behaviour can be deduced from the Helmholtz free energy per particle, $f=f(\rho, T)=F / N \varepsilon_{0}$, of Eq. (8) where $\rho$ is the number density, $\rho=N / V$. From $f=f(\rho, T)$ we can obtain the pressure, $p=p(\rho, T)$, and the chemical potential, $\mu=\mu(\rho, T)$, by using the following well known thermodynamic relations [2]:

$$
p=\rho^{2} \frac{\partial f}{\partial \rho} ; \mu=\frac{\partial}{\partial \rho}(\rho f) ;
$$

and, knowing $p$ and $\mu$ we can find the coexisting densities $\left(\rho_{1}, \rho_{2}\right)$ of phase 1 and phase 2 by solving, for each $T$, the following two-phase equilibrium conditions [2]:

$$
\begin{array}{r}
p_{1}\left(\rho_{1}, T\right)=p_{2}\left(\rho_{2}, T\right), \\
\mu_{1}\left(\rho_{1}, T\right)=\mu_{2}\left(\rho_{2}, T\right),
\end{array}
$$

where $P_{i}, \mu_{i}$ denote the value of $P, \mu$ evaluated for phase $i=(1,2)$.

When more than two phases are present for the same T, Equations (23) - (24) are solved for each pair of coexisting phases, and the thermodynamically stable phase transitions are separated from the thermodynamically metastable phase transitions by constructing the convex envelope to the free energy with the aid of Maxwell's double tangent construction [33].

\subsection{The fluid phases}

In a first step we will consider only fluid phases and disregard the solid phases altogether. This is an often adopted attitude when, like here, the central question concerns the possible existence of a (nematic) liquid phase. As already stated above, such an attitude is hampered by the fact that several of the fluid-fluid transitions which can be found in this way may still be pre-empted by some of the fluid-solid transitions not explicitly considered here. In other words, many fluid phases, although mechanically stable, can still be thermodynamically metastable.

In the present context we will consider the competition between two types of uniform fluid phases: the isotropic (I) fluid phase (phase 1) without orientation order $(m=0)$, and the nematic $(\mathrm{N})$ fluid phase (phase 2 ) for which the spin variables are, on average, aligned along some director $(m \neq 0)$.

From Eqs. (8) and (23) the pressure of the fluid phase can be written as

$$
\frac{p}{\rho \varepsilon_{1}}=\frac{t}{\alpha(\rho)}-\phi_{1}-\gamma \phi_{2} m^{2}
$$


where $\alpha(\rho)$ is given by (12) and $\phi_{l}(l=1,2)$ by (16). The orientation order parameter, $m=m(\rho, t)$, is obtained by solving equations (9) and (10), yielding hence $p=p(\rho, t)$, as needed for Eq. (23), and similarly for $\mu=\mu(\rho, t)$ by using Eq. (24).

According to the value of $\Gamma^{*}=\gamma\left(n_{1}-3\right) /\left(n_{2}-3\right)$ there are two topologically distinct types of phase diagrams (see [32]). For weakly anisotropic fluids, $0<\Gamma^{*}<1.0863$, all the phase diagrams have a isotropic gas (IG)-isotropic liquid (IL) and a IL-nematic fluid (NF) transition. For temperatures below the triple point temperature $t_{t}$ there is a single firstorder transition, namely a IG-NF transition. Above $t_{t}$ there are two first-order transitions, namely a IG -IL transition ending at the critical point and a first-order IL-NF transition. For $t>t_{c}$ there is only a first-order IF-NF transition. For stronger nematic anisotropies, $\Gamma^{*}>1.0863$, the critical point becomes metastable and the phase diagram contains only a IF-NF transition.

\subsection{The solid phase}

In order to compute the total phase diagram (where both fluid and solid phases are considered), and to show hence the stability of the nematic liquid we first introduce the solid phases.

It is seen from the latter section that for $\Gamma^{*}>1.0863$, the IF-NF transition pre-empts the IG-IL transition. In a similar manner the fluid (F)-solid (S) transition can still preempts this IF-NF transition. To answer the question whether a (stable) ferromagnetic fluid phase is present in the phase diagram, it is thus essential to include both fluid and solid phases. Before considering the complete phase diagram, we devote this section to the case where both phases in Eqs. (23) and (24) are solid phases.

The stable lattice structure (solid phase) is seen to be a compact lattice such as facecentred-cubic or hexagonal close packed.

In this paper we take this lattice structure to be of the face-centred-cubic $(f c c)$ type, because, in addition to its higher symmetry (see tab.1), exact computer simulations and theoretical work on mono-disperse colloidal hard spheres indicate that the stable crystal structure is $f c c[34,35]$.

Table 1 shows the number of nearest neighbours for $f c c$. Here $n$ and $N$ represent, respectively, the number of the shell and the number of particles in this shell.

Table 1.

\begin{tabular}{ll}
\hline$n$ & $N(f c c)$ \\
\hline 1 & 12 \\
2 & 0 \\
3 & 6 \\
4 & 0 \\
5 & 24 \\
\hline
\end{tabular}


For a $f_{c c}$ lattice, the maximum value of $\eta_{c p}$ is

$\eta_{c p}=\frac{\pi}{3 \sqrt{2}} \cong 0.74$

In fact, considering an unit cell for $f_{c c}$ lattice of volume $V=a^{3}$. At closing packing ( $a=\sqrt{2} \sigma$ ), the volume becomes

$$
V=V_{c p}=a^{3}=2 \sqrt{2} \sigma^{3},
$$

where $\sigma$ is the diameter of the particle.

Moreover, the volume at closing packing can also be written as

$$
V=\frac{4}{\rho_{c p}},
$$

where 4 is the number of particles per unit cell and $\rho_{c p}$ the density at closing packing.

By combining Eqs. (27) and (28) one obtains

$$
\eta_{c p}=\frac{\pi}{6} \rho_{c p} \sigma^{3}=\frac{\pi}{3 \sqrt{2}} \cong 0.74
$$

In this section we will consider thus the competition between two types of uniform solid phases: the isotropic solid phase $(\mathrm{m}=0)$, and the nematic solid phase $(m \neq 0)$.

From Eqs. (8) and (22), the pressure can be written as

$$
\frac{p}{\rho \varepsilon_{1}}=\frac{t}{1-\left(\rho / \rho_{c p}\right)^{1 / 3}}-\frac{n_{1}}{6} \alpha_{n_{1}}\left(\rho / \rho_{c p}\right)^{n_{1} / 3}-\frac{n_{2}}{6} \alpha_{n_{2}}\left(\rho / \rho_{c p}\right)^{n_{2} / 3} \gamma m^{2}
$$

where $\frac{\pi}{6} \sigma^{3} \rho_{c p}=0.74$ for a $f c c$ structure and $\alpha_{n_{i}}$ is the Madelung constant of the $f c c$ lattice for an inverse power potential of index $n_{i} \mathrm{i}=(1,2)$.

As can be seen from Eq. (21), $\alpha_{n_{i}}$ tends to 12, the number of nearest neighbours of the fcc lattice, for large $n_{i}$ values.

Contrary to the fluid case, the phase diagram of the solid phase depends explicitly on the three parameters $\left\{\gamma, n_{1}, n_{2}\right\}$. In the following we will consider the long-ranged isotropic interactions $\left(n_{1}=6\right)$ and long-ranged nematic interactions $\left(n_{2}=6\right)$. Depending on the value of these parameters, two topologically distinct phase diagrams are considered (see Fig.2). For $0<\gamma<0.647$ all phase diagrams have an isotropic expanded solid (IES) (low density solid) - isotropic condensed solid (ICS) (solid of the same structure but of a higher density) and an isotropic solid (IS) - nematic solid (NS) transitions (see Fig.2.a). For $\gamma>0.647$ only the IS-NS transitions survive (see Fig.2.b). The critical point (open square in Fig.2.a) is determined by solving equations:

$$
\frac{\partial p}{\partial \eta}=0 \quad ; \quad \frac{\partial^{2} p}{\partial \eta^{2}}=0
$$


One finds

$$
t_{c}^{\prime}=n_{1}\left(1+\frac{n_{1}}{3}\right) \alpha_{n_{1}} y^{n_{1}} \frac{(1-y)^{2}}{\left(1-\frac{2}{3} y\right)}, \quad \eta_{c}^{\prime}=y^{3} \frac{\pi}{3 \sqrt{2}}
$$

with y given by

$$
y \equiv\left(\frac{5 n_{1}+4}{4 n_{1}-4}\right)-\left[\left(\frac{5 n_{1}+4}{4 n_{1}-4}\right)^{2}-\left(\frac{3 n_{1}}{2 n_{1}-2}\right)\right]^{1 / 2}
$$
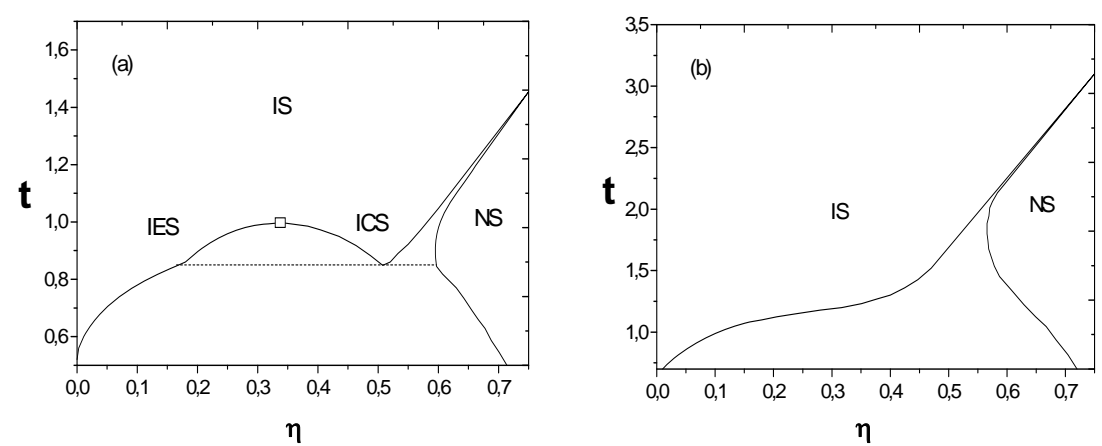

Fig. 2. The two topologically distinct types of phase diagrams in the reduced temperature $(t=$ $\left.k_{\mathrm{B}} T / \varepsilon_{1}\right)$-reduced density $\left(\eta=\frac{\pi \rho \sigma^{3}}{6}\right)$ plane as obtained from the free energy (8) when only solid phases are considered. (a) The open square marks the solid-solid critical point. (The case shown corresponds to $n_{1}=n_{2}=6$, and $\gamma=0.5$ ). (b) The critical point has disappeared so that there are only two phases. (The case shown corresponds to $n_{1}=n_{2}=6$, and $\gamma=1$ ). The triple point at which the three phases NS, ICS and IES coexist disappears for $\gamma=0.647$ ).

\subsection{The complete phase diagram}

In order to obtain a complete phase diagram we now consider the case where in Eqs. (23) and (24) phase 1 is a fluid phase and phase 2 a solid phase, and combine these fluid-solid transitions with the fluid-fluid and solid-solid transitions already considered in the two previous sections. Stated differently, the fluid-fluid transitions obtained from Eqs.(23) and (24) correspond to double tangent constructions on the free energy (8) when the latter is evaluated for the fluid phase, and similarly for the solid-solid transitions when Eq. (8) is evaluated for the solid phase, whereas the fluid-solid transitions correspond to double tangent constructions between the free energy of the fluid and that of the solid. When more than one double tangent construction is possible, the complete phase diagram is obtained from the convex envelope to the free energies. Since the solid-solid transitions do depend on the three parameters $\left\{\gamma, n_{1}, n_{2}\right\}$ separately, so will the complete phase 
diagrams. We know from $[4,9,15,32]$ that when $\gamma=0$ there are three topologically distinct types of phase diagrams according to the value of $n_{1}\left(n_{2}\right.$ is irrelevant when $\left.\gamma=0\right)$.

For long-ranged interactions $\left(3<n_{1}<7.6\right)$ the phase diagram exhibits the $\left(t_{c}, \eta_{c}\right)$ critical point (see [32]), for intermediate-ranged interactions $\left(7.6<n_{1}<67\right)$ there is no critical point, whereas for short-ranged interactions $\left(n_{1}>67\right)$ it exhibits the $\left(t_{c}^{\prime}, \eta_{c}^{\prime}\right)$ of Eq. (32). Here we consider the long $\left(\left(n_{1}=6\right)\right.$-long $\left(n_{2}=6\right)$ case. Depending on the value of $\gamma$ three types of phase diagrams can occur. For small value of $\gamma$ the phase diagram contains four phases, namely IG, IL, IS, and NS and exhibits a triple points (where IG, IL, and NS are present) (see Fig.3.a). For larger values of $\gamma$ the isotropic IG-IL transition disappears, and only a IF-NS survives at low temperatures (see Fig.3.b). For even larger value of $\gamma$ a first-order IL-NF transition appears with the presence of two triple point temperatures (see Fig.3.c).
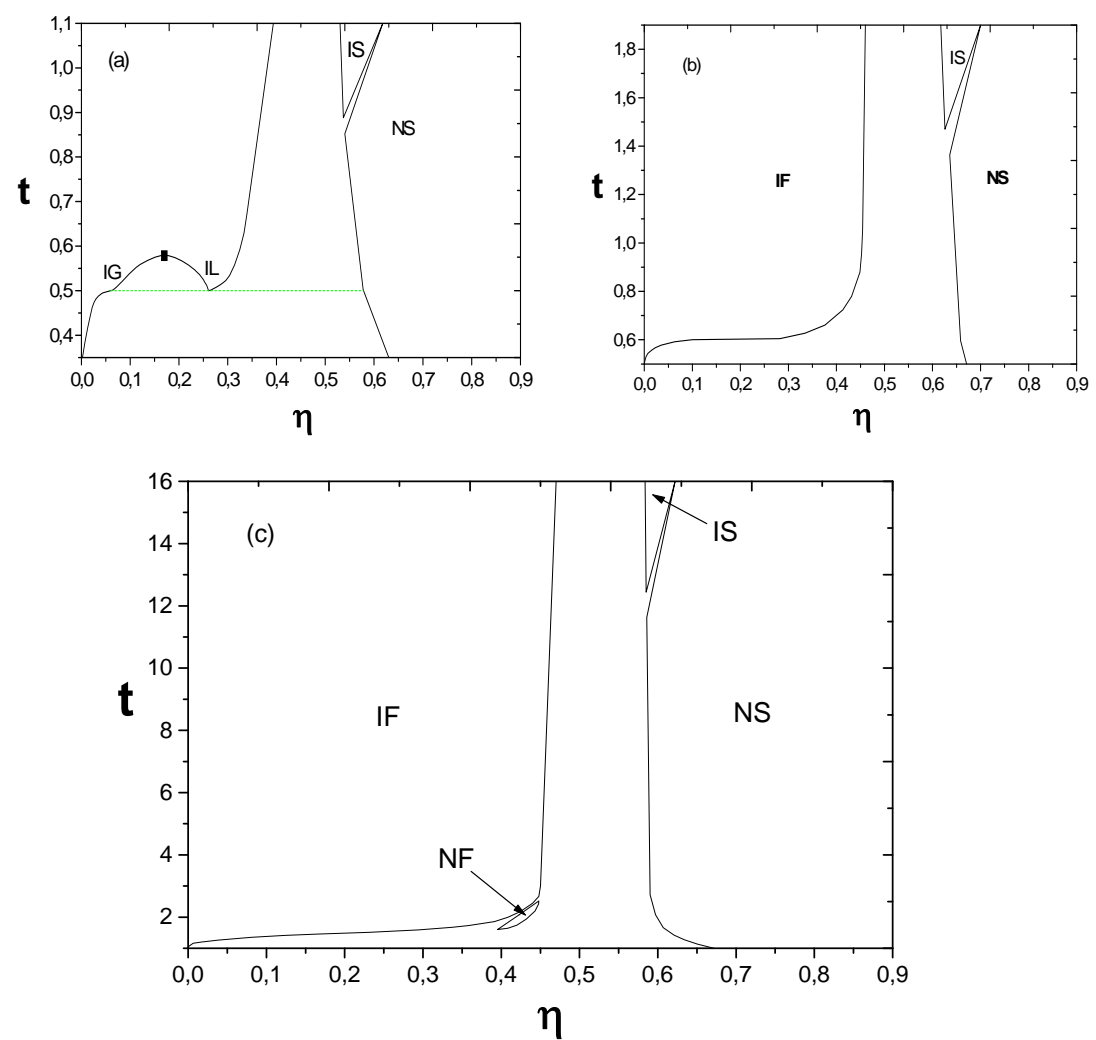

Fig. 3. Complete phase diagram, for both fluid and solid phases. The case shown corresponds to $n_{1}=$ $n_{2}=6$. (a) There are four phases: IG, IL, IS and NS with a critical point (filled square). (The case shown corresponds to $\gamma=0.3$ ). (b) the IG-IL transition of (a) has become metastable. (The case shown corresponds to $\gamma=0.6$ ). (c) The NF phase becomes now stable and we have two IF-NF-NS triple lines which confine the $\mathrm{N}$-phase to a small pocket of the $t-\eta$ plane. (The case shown corresponds to $\gamma=3$ ). 


\section{Conclusion}

The presence of attraction is a necessary condition for the appearance of a liquid phase in the phase diagram. As has been shown in [4,5], it is however not a sufficient condition. Indeed, in the case of a long ranged, weak attractive potential one recovers the van der waals model which shows a first-order liquid-vapour transition ending in a critical point. For a system with a shorter interaction range the liquid phase becomes metastable. In the present work, we have investigated the case of complex fluids composed of spherical molecules endowed with both translational (described in terms of the position of the centre of mass of the molecule) and orientational (described in terms of a classical spin variable) degrees of freedom.

We have studied the phase diagram of a system of particles interacting via both nematic and isotropic interactions by using the extended vdW theory. We have considered the long- $\left(n_{1}=6\right)$-long $\left(n_{2}=6\right)$ case. We have found that the topology of the phase diagrams depends sensitively on the relative strengths of the isotropic and nematic interactions. We have found that, (contrary to what happens when the solid phases are not taken into account [32]), the nematic fluid is stable only for large values of the strength of the nematic interactions. We have shown also that the nematic fluid is thermodynamically stable only in a small domain of the temperature-density plane contained between two triple lines.

\section{Acknowledgments}

We gratefully acknowledge enlightening discussions with Prof. M. Baus (ULB), and Z. El Allali for his help in computation.

\section{References}

1. D. L. Goodstein, States of matter, (Dover, New York, 1985).

2. D. A. Young, Phase Diagrams of the Elements, (University of California Press, Berkely, 1991).

3. J. P. Hansen and I. R. McDonald, Theory of Simple Liquids, $1^{\text {st }}$ ed. (Academic, London, 1976).

4. C. F. Tejero, A. Daanoun, H. N. W. Lekkerkerkr, and M. Baus, Phys. Rev. E 51, 558 (1995). doi:10.1103/PhysRevE.51.558

5. C. Rascon, G. Navascues, and L. Mederos, Phys. Rev. B 51, 14799 (1995). doi:10.1103/PhysRevB.51.14899

6. P. I. C.Teixeira, Mol. Phys. 92, 167 (1997).

7. R. Achrayah and M. Baus, Phys. Rev. E 57, 4361 (1998). doi:10.1103/PhysRevE.57.4361

8. C. F. Tejero and M. Baus, Phys. Rev. E 57, 4821 (1998). doi:10.1103/PhysRevE.57.4821

9. A. Daanoun , C.F. Tejero, and M. Baus, Phys. Rev. E 50, 2913 (1994). doi:10.1103/PhysRevE.50.2913

10. M. Baus and R. Achrayah, J. Phys. Condens. Matter 8. 9633 (1996). doi:10.1088/0953-8984/8/47/075

11. T. Caussaert and M. Baus, Phys. Rev. E 52, 862 (1995). doi:10.1103/PhysRevE.52.862 
12. G. Monaco, S. Falconi, W.A. Crichton, M. Mezouar, Phys. Rev. Lett. 90, 255701 (2003). doi:10.1103/PhysRevLett.90.255701

13. S. V. Buldyrev and H. E. Stanley, Physica A 330, 124 (2003). doi:10.1016/j.physa.2003.08.003

14. A. Daanoun, Eur. Phys. J. B. 531, 61 (2006). doi:10.1140/epjb/e2006-00344-1

15. A. Oukouiss and M. Baus, Phys. Rev. E 55, 7242 (1997). doi:10.1103/PhysRevE.55.7242

16. A. Oukouiss and M. Baus, J. Chem. Phys. 109, 6157 (1998). doi:10.1063/1.477243

17. J. A. Cuesta, C. F. Tejero, and M. Baus, Phys. Rev. A 45, 7395 (1992). doi:10.1103/PhysRevA.45.7395

18. P. G. De Gennes, The physics of liquid crystals (Oxford University Press, Clarendon, 1974).

19. P. M. Chaikin and T.C. Lubensky Prinxipes of condensed matter physics (Cambridge University Press, Cambridge, 1995).

20. L. Onsager, Proc. N. Y. Acad. Sci. 51, 627 (1949).

21. J. L. Colot, X-G. Wu, H. Xu, and M. Baus, Phys. Rev. A 38, 2022 (1998). doi:10.1103/PhysRevA.38.2022

22. D. Frenkel, Mol. Phys. 60, 1 (1987). doi:10.1080/00268978700100011

23. D. Frenkel, H. N. W. Lekkerkerker, and A.Stroobants, Nature 332, 822 (1988). doi:10.1038/332822a0

24. D. Frenkel, J. Phys. Chem. 92, 3280 (1988). doi:10.1021/j100322a042

25. J. A. C. Veerman and D. Frenkel, Phys.Rev. A 41, 3237 (1990). doi:10.1103/PhysRevA.41.3237

26. S. C. McGrother, D. C.Williamson, and G. Jackson, J. Chem. Phys. 104, 6755 (1996). doi:10.1063/1.471343

27. M. J. Blair and G. N. Patey, Phys. Rev. E57, 5682

(1997). doi:10.1103/PhysRevE.57.5682

28. P. Mishra, Y.Singh, Phys. Rev. Lett. 97, 177801 (2006). doi:10.1103/PhysRevLett.97.177801

29. P. Mishra, S. Lata Singh, J. Ram, and Y. Singh, J. Chem. Phys. 127, 044905 (2007). doi:10.1063/1.2752170

30. N. H. Phoung and F. Schmid, J. Chem. Phys. 119, 1214 (2003). doi:10.1063/1.1577322

31. E. Lomba, C. Martin, and N. Almarza, Phys. Rev. E74, 021503 (2006). doi:10.1103/PhysRevE.74.021503

32. A. Oukouiss and A. Daanoun J. Sci. Res. 1 (3), 409 (2009). doi:10.3329/jsr.v1i3.2595

33. R. T. De Hoof, Thermodynamics in Material Science (McGraw-Hill, New York 1993).

34. D. Frenkel and A. J. C. Ladd, J. Chem. Phys. 81, 3188 (1994). doi:10.1063/1.448024

35. K. W. Kratky, J. Chem. Phys. 57, 167 (1981). doi:10.1016/0301-0104(81)80031-6 